\title{
Investigation of faint galactic carbon stars from the First Byurakan Spectral Sky Survey. IV. GAIA DR2 data.
}

\author{
Kostandyan G. R.* \\ V .A. Ambartsumian Byurakan Astrophysical Observatory, Armenia
}

\begin{abstract}
The second Gaia data release (Gaia DR2) data are used to analyze and estimate a some important parameters for 127 carbon (C) type stars (56 are late N - type Asymptotic Giant Branch (AGB) C stars, 71 are early - type CH giants) detected on the First Byurakan Spectral Sky Survey (FBS) low - resolution (lr) spectral plates. Gaia DR2 G broad band magnitudes are in the range $9.4 \mathrm{mag}<\mathrm{G}<18.2 \mathrm{mag}$. for FBS $\mathrm{C}$ stars. Radial velocities (RV) is available for $75 \mathrm{C}$ stars out of 127 . For 9 objects RV is greater than $200 \mathrm{~km} / \mathrm{s}$. Absolute magnitudes in $\mathrm{V}$ band are estimated for 18 FBS C stars, having luminosity data, from which 17 are $\mathrm{CH}$ giants. They are in the range between $-3.5 \leq \mathrm{M}_{V}<+0.5 \mathrm{mag}$. For FBS 1918+869 absolute magnitude $\mathrm{M}_{V}=-3.4( \pm 0.2) \mathrm{mag}$, which is typical for $\mathrm{N}$ type AGB C stars. Having distance estimations, the Hertzsprung - Russell diagram (HRD, or color - absolute magnitude diagram) was constructed for $\mathrm{C}$ stars. All FBS detected C stars are giants and AGB stars in the Galactic Halo. They are not far than $14 \mathrm{kpc}$ from the Sun and $8 \mathrm{kpc}$ from the Galactic plane.
\end{abstract}

Keywords: carbon stars: surveys: late - type stars

\section{Introduction}

The First Byurakan Survey (FBS, known also as a Markarian survey ) is an objective - prism low - resolution (lr) survey. It is performed with the Byurakan Observatory $1 \mathrm{~m}$ Schmidt telescope, which covers about 17.000 sq. deg. of the Northern sky and part of the Southern sky at high Galactic latitudes defined by $\delta>-15^{\circ}$ and IbI $>15^{\circ}$. The FBS was originally conducted for galaxies with ultraviolet excess (UVX, Markarian et al., 1989). Since 1990s, the lr plates of the FBS was used to select comparatively faint (fainter than 12 mag. in visual) late - type stars (LTS, M and carbon (C) stars) at high latitudes. The large spectral range of the FBS $(\lambda 3400-6900 \AA)$ is well suited to identify cool M - type or C - type stars. C stars can be identified through the presence of Swan bands of $\mathrm{C}_{2}$ molecule at 4737, 5165, and $5636 \AA$ (N - type $\mathrm{C}$ stars). Several objects showing the $\mathrm{C}_{2}$ band-head at $4382 \AA$ are early - type $\mathrm{C}$ stars ( $\mathrm{R}$ or $\mathrm{CH}$ type stars). M - type stars can easily be distinguished because of the titanium oxide ( $\mathrm{TiO}$ ) molecule absorption bands at 4584, 4762, 4954, 5167, 5500 and $6200 \AA$ (Gigoyan \& Mickaelian, 2012). The eye - piece search (with magnification 15x) near 2000 FBS Ir plates resulted to discovery 1045 new LTS. On the base of this selection the "Revised And Updated Catalogue of the First Byurakan Survey of LTS" was generated (Gigoyan \& Mickaelian, 2012). Now the entire plate set of the FBS has been digitized (1874 plates for 1139 fields) leading to the Digitized First Byurakan Survey (DFBS) (Mickaelian et al., 2007) ( online at http://byurakan.phys.uniroma.it or http://www.ia2-byurakan.oats.it/). Later, all DFBS lr spectral plates are analysed with help of standard image analysis Softwares (FITSView and SAOImage ds9). The advantages using these Softwares for selecting faint LTS on DFBS plates are described more detail in Gigoyan et al. (2012). "The Second Revised And Updated Version of the FBS LTS Catalogue", containing comprehensive data for 1471 new objects, is available at CDS, SIMBAD VizieR data base (Gigoyan et al., 2019).

${ }^{*}$ kgayane@gmail.com 

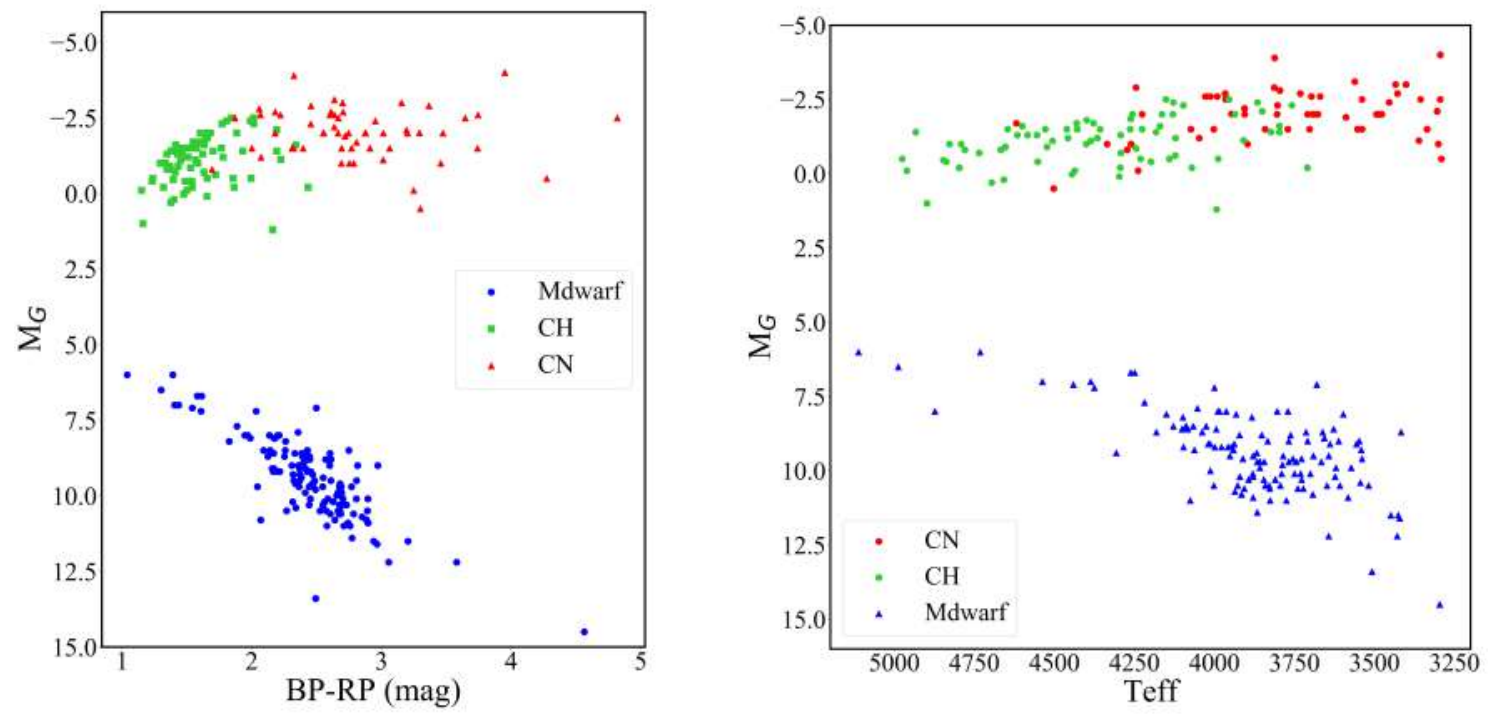

Figure 1. Hertzsprung - Russell diagramms for 127 FBS C stars

The goal of this paper is further exploitation of the Gaia Data Release 2 (DR2) (Gaia Collaboration et al., 2018) high accurate photometric and astrometric data, also the distance estimations, presented in catalogue of Bailer-Jones et al. (2018b), to estimate important parameters, such as absolute magnitudes and distribution in our Galaxy for carbon stars, selected on the lr FBS survey plates, and presented in the "Second and Updated Version of the FBS Late-Type Stars Catalogue (Gigoyan et al., 2019).

In Section 2 we describe Gaia DR2 data for FBS Carbon stars and some important physical parameter estimations. We discuss in Section 3 their Galactic distribution. Finally we conclude in Section 4.

\section{FBS carbon stars. Gaia DR2 data and some important parameters}

Gaia second data release (DR2), consists astrometry, photometry, radial velocities, effective temperatures, information on astrophysical parameters and variability, for sources brighter than $\mathrm{G}=21.0$ magnitude (approximately 1.7 billion sources, Gaia Collaboration et al., 2018). All FBS discovered C stars was cross - correlated with the Gaia DR2 catalogue sources (SIMBAD CDS VizieR Catalog I/345/gaia2). The search was made in catalogue with a search radius $5^{\prime \prime}$ around their position. Their $\mathrm{G}$ broad - band photometric data values is in the range 9.4 mag. $<\mathrm{G}<18.2$ mag., and effective temperatures between $3291.00 \mathrm{~K}<$ Teff $<5000 \mathrm{~K}$.

\subsection{Colours, luminosities, and other characteristics}

In Gaia DR2 data base the radiuses and luminosities (in Solar units)is presented for 18 FBS C stars only (out of 127). We estimate the absolute magnitudes (in $\mathrm{V}$ - band) for 18 objects adopting that $\mathrm{M}_{V \odot}=+4.81 \mathrm{mag}$ for Sun. Figure 1 show positions on colour - absolute MG magnitude (Gaia DR2 G - band absolute magnitude) and $\mathrm{T}_{\text {eff }}$ versus absolute magnitude (Hertzsprung - Russell) diagram for 127 FBS C stars, also for near 150 M dwarfs from the "FBS Late - Type Stars Catalogue" (Gigoyan et al., 2019). All N - type AGB stars are distributed in the brightest region, where Long - Period Variables (Miras, Semi - Regular variables, slow irregular variables, and small - amplitude red giant) are located and Figure 2 and 3 by Gaia Collaboration et al. (2019). Absolute G - band magnitude was estimated via the usual equation (Gaia Collaboration et al., 2019): 


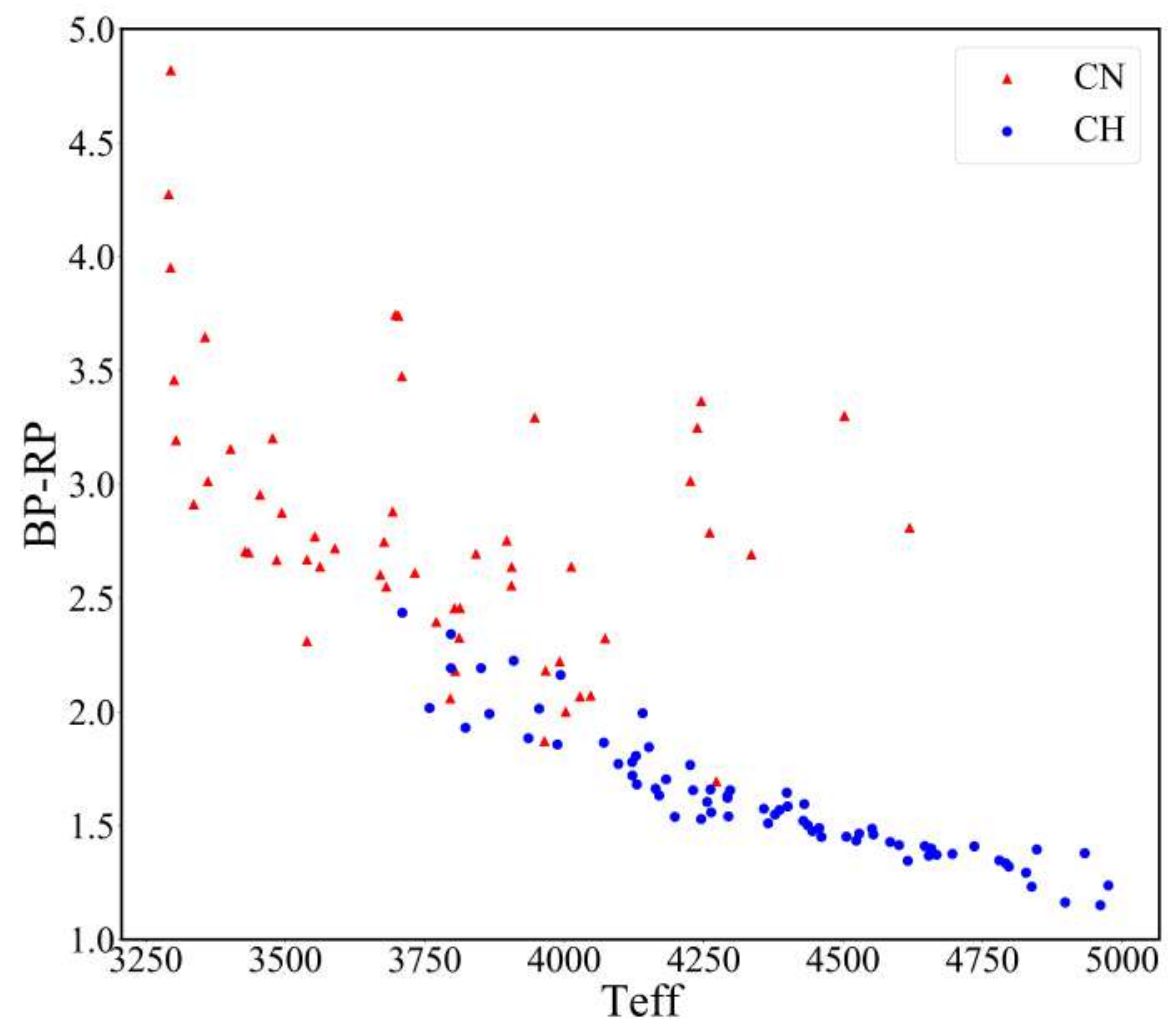

Figure 2. Gaia DR2 color - temperature relation for 127 FBS C stars.

$$
M_{G}=G-5 \log r+5-A_{G},
$$

As we can see, FBS C stars occupy region on the HR diagram, where red giants and AGB stars are located. Figure 2 shows Gaia DR2 color - temperature relation for 127 FBS C stars. $11 \mathrm{~N}$ - type AGB stars are far from the line of distribution of stars. For all of them 2MASS (Two Micron All - Sky Survey, Skrutskie et al., 2006) J $-\mathrm{K}_{S}>2.5 \mathrm{mag}$. Most probably there are thick gas-dust envelopes around these stars. Based on K- [12] and J - K colors indices mass-loss rates were estimated for 3 of them (Gigoyan et al., 2017). Three out of $11 \mathrm{~N}$ - type AGB stars show double - peaked SED Gigoyan et al. (2017), indicating the existence of the envelopes around them. We note also, that the effective temperatures, determined from the photometric data for $\mathrm{CH}$ - type FBS $\mathrm{C}$ stars (Gigoyan et al., 2015), are in the range of uncertainties, which gives Gaia DR2 catalogue (SIMBAD VizieR Catalog I/345/gaia2, Gaia Collaboration et al., 2018).

\section{Distances}

Distance estimations for all detected FBS C stars is presented in catalogue by Bailer-Jones et al. (2018a), which is based on Gaia DR2 parallaxes. In this catalogue distance estimation for more than 1.33 billion stars are reported. In the first paper of this series (Gigoyan et al., 2014) distances are estimated based on revised Period - Luminosity (PL) relations for N - type AGB C stars. According to Bailer-Jones catalogue data, the distances are in the range $735.64 \mathrm{pc}<\mathrm{d}<14133.43 \mathrm{pc}$ (FBS $0018+213, \mathrm{~L}=82.779 \mathrm{~L}_{\odot}, \mathrm{RV}=-13.87( \pm 1.54) \mathrm{km} / \mathrm{s}$, and FBS $1811+462, \mathrm{RV}=-164.8( \pm 0.65)$ $\mathrm{km} / \mathrm{s})$. Both stars are $\mathrm{CH}-$ giants. The hight $(\mathrm{Z})$ above Galactic plane for FBS $0018+213$ is $\approx 480$ pc, and for FBS $1811+462$ is $\approx 6.1 \mathrm{kpc}$ consequently. 
Figure 3 shows spatial distribution (Galactic longitude versus Galactic latitude) for all FBS C stars. Very little tendency is observable for $\mathrm{CH}$ giants distribution at high Galactic region.

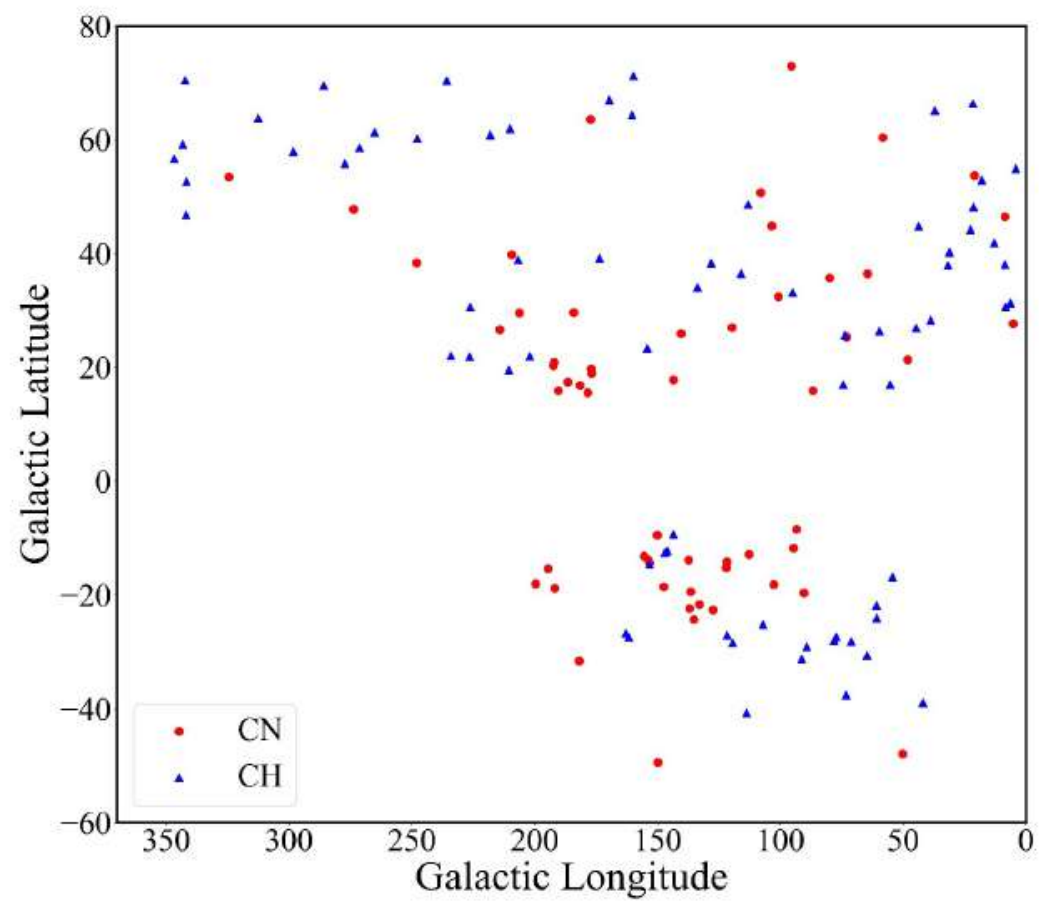

Figure 3. Spatial distribution for 127 FBS C stars.

\section{Discussion and conclusion}

Carbon stars, are excellent kinematics tracers of galaxies. They can also be served as visible standard candles for galaxies. Meanwhile, many problems remain not solved with this classes of objects, particularly estimation of absolute magnitudes for early - type of C stars, which are warm objects( $\mathrm{R}$ - type stars, $\mathrm{CH}$ - giants, and dwarf Carbon stars (dC)). Late $\mathrm{N}$ - type carbon stars are cool and luminous AGB stars and they follow Period-Luminosity (PL) relations. In this paper, the second Gaia Data Release 2 (DR2) high-precision astrometric and photometric data together with the CSS data base are used to analyse and estimate some very important physical characteristics for a limited number of carbon stars, discovered on the FBS plates, with a goal to clarify the nature of this objects at high latitudes. We study 127 FBS C stars from the light of Gaia, from which 56 are N - type AGB stars and 71 are $\mathrm{CH}$ - type giants. Having distance estimations, the Hertzsprung - Russell diagram (HRD, or color - absolute magnitude diagram) was constructed for C stars. All FBS detected C stars are giants and AGB stars in the Galactic Halo. They are not far than $14 \mathrm{kpc}$ from the Sun and $8 \mathrm{kpc}$ from the Galactic plane. Their Gaia DR2 G broad - band absolute magnitudes are estimated which is the range $+1.0 \leq \mathrm{G} \leq-4.0$ mag. For 18 FBS $\mathrm{C}$ stars absolute $\mathrm{V}$ - band magnitudes are estimated, having luminosities in DR2 data base, from which 17 are $\mathrm{CH}$ giants. They are in the range between $-3.45( \pm 0.2) \leq \mathrm{M}_{V}<+0.5( \pm 0.1)$ mag. For FBS 1918+869 absolute magnitude $\mathrm{M}_{V}=-3.4( \pm 0.2) \mathrm{mag}$, which is typical for $\mathrm{N}$ - type AGB C stars. Radial velocities is available for $75 \mathrm{C}$ stars out of 127 . For 9 objects RV values is greater than $200 \mathrm{~km} / \mathrm{s}$. These stars are supplements to the objects with high radial velocity data discovered recently based on Gaia DR2 data base. We note that all these objects are Galactic Halo objects and they do not trace the Sagittarius (Sgr) dwarf spheroidal galaxy streams and their origin is unclear.

Meanwhile, it is worth to mention three FBS CH type giants among the 127 FBS C stars, for which 
Bailer-Jones catalogue gives distances more than $13 \mathrm{kpc}$ from the Sun. They are FBS 1629+156( r $=13.04 \mathrm{kpc}$,$) , FBS 1811+462(\mathrm{r}=14.133 \mathrm{kpc}, \mathrm{RV}=-164.8 \mathrm{~km} / \mathrm{s})$, and FBS $1454+792(\mathrm{r}=13.487$ $\mathrm{kpc}, \mathrm{RV}=-217.9 \mathrm{~km} / \mathrm{s}$ ). According to the Figure 15 in Huxor \& Grebel (2015) the star FBS $1454+792$ and FBS $1811+462$ can be originated from the Sgr leading arm.

For FBS $1629+156$ there are no RV information in Gaia DR2 data base. The object FBS $1811+462$ show CSS phase-dependence light curve, which is typical for RS CVn type variable stars. In NSVS (Northern Sky Variability Survey, http://skydot.lanl.gov/) data base the object FBS 1629+156 can be also classified as RS CVn variable with amplitude $\triangle \mathrm{m} \approx 0.4 \mathrm{mag}$. In CSS data base this object do not show significant variability. Concerning to objects FBS $1454+792$, this object is out of the CSS field and in NSVS data base do not show any variability.

We note also, that many - sided investigations which is based on Gaia DR2 for M - type giants and dwarfs from the "Second Revised and Updated Catalogue Of the FBS LTS" is in progress and will be submitted for publication very soon.

\section{References}

Bailer-Jones C. A. L., Rybizki J., Fouesneau M., Mantelet G., Andrae R., 2018a, VizieR Online Data Catalog, p. I/347

Bailer-Jones C. A. L., Rybizki J., Andrae R., Fouesneau M., 2018b, A\&A, 616, A37

Gaia Collaboration et al., 2018, A\&A, 616, A1

Gaia Collaboration et al., 2019, A\&A, 623, A110

Gigoyan K. S., Mickaelian A. M., 2012, MNRAS, 419, 3346

Gigoyan K. S., Russeil D., Mickaelian A. M., Sarkissian A., Avtandilyan M. G., 2012, A\&A, 544, A95

Gigoyan K. S., Sarkissian A., Russeil D., Mauron N., Kostandyan G., Vartanian R., Abrahamyan H. V., Paronyan G. M., 2014, Astrophysics, 57, 510

Gigoyan K. S., Sarkissian A., Russeil D., Mauron N., Kostandyan G., Vartanian R., Abrahamyan H. V., Paronyan G. M., 2015, Astrophysics, 58, 369

Gigoyan K. S., Sarkissian A., Rossi C., Russeil D., Kostandyan G., Calabresi M., Zamkotsian F., Meftah M., 2017, Astrophysics, 60, 70

Gigoyan K. S., Mickaelian A. M., Kostandyan G. R., 2019, MNRAS, 489, 2030

Huxor A. P., Grebel E. K., 2015, MNRAS, 453, 2653

Markarian B. E., Lipovetsky V. A., Stepanian J. A., Erastova L. K., Shapovalova A. I., 1989, Soobshcheniya Spetsial'noj Astrofizicheskoj Observatorii, 62, 5

Mickaelian A. M., et al., 2007, A\&A, 464, 1177

Skrutskie M. F., et al., 2006, AJ, 131, 1163 